PROCEEDINGS OF THE

AMERICAN MATHEMATICAL SOCIETY

Volume 124, Number 6, June 1996

\title{
THE EXTENSIONS OF THE FERENC MÓRICZ THEOREMS
}

\author{
S. S. BHATIA AND BABU RAM
}

(Communicated by J. Marshall Ash)

\begin{abstract}
We study the integrability of the $r$ times differentiated complex trigonometric series using modified trigonometric sums and obtain a new necessary and sufficient condition for $L^{1}$-convergence of the $r$ th derivative of the Fourier series. Some results of F. Móricz are deduced as corollaries.
\end{abstract}

\section{INTRODUCTION}

A complex null sequence $\left\{c_{k}\right\}$ satisfying $\sum_{k=1}^{\infty}\left|\Delta\left(c_{k}-c_{-k}\right)\right| \log k<\infty$ is called weakly even and is denoted by $\left\{c_{k}\right\} \in W$. If a null sequence $\left\{c_{k}\right\}$ satisfies $\sum_{k=1}^{\infty}\left|\Delta\left(c_{k}-c_{-k}\right)\right| k^{r} \log k<\infty$ for some $r=0,1,2, \ldots$, then we write that $\left\{c_{k}\right\} \in W_{r}$, where $W_{0}=W$. If there exists $\beta>0$ such that $n^{-\beta} a_{n} \downarrow 0$, then the sequence $\left\{a_{n}\right\}$ is called a quasi-monotone sequence and is denoted by $a_{n} \curvearrowleft 0$.

The partial sums of the complex trigonometric series $\sum_{|n| \leq \infty} c_{n} e^{\text {int }}$ will be denoted by $s_{n}(c, t)=\sum_{|k| \leq n} c_{k} e^{i k t}, t \in T=\mathbb{R} / 2 \pi Z$. If a trigonometric series is the Fourier series of some $f \in L^{1}$, we shall write $c_{n}=\hat{f}(n)$ for all $n$ and $s_{n}(c, t)=s_{n}(f, t)=s_{n}(f)$.

Let $D_{n}(t)$ and $\widetilde{D}_{n}(t)$ denote the Dirichlet and the conjugate Dirichlet kernel respectively. Let $E_{n}(t)=\sum_{k=0}^{n} e^{i k t}$ and $E_{-n}(t)=\sum_{k=1}^{n} e^{-i k t}$. Then the $r$ th derivatives $D_{n}^{(r)}(t)$ and $\widetilde{D}_{n}^{(r)}(t)$ of $D_{n}(t)$ and $\widetilde{D}_{n}(t)$ can be written as

$$
\begin{aligned}
D_{n}^{(r)}(t) & =E_{n}^{(r)}(t)+E_{-n}^{(r)}(t), \\
i \widetilde{D}_{n}^{(r)}(t) & =E_{n}^{(r)}(t)-E_{-n}^{(r)}(t),
\end{aligned}
$$

where $E_{n}^{(r)}(t)$ denotes the $r$ th derivative of $E_{n}(t)$.

Č. V. Stanojević and V. B. Stanojević [5] introduced the following modified complex trigonometric sum:

$$
u_{n}(c, t)=s_{n}(c, t)-\left(c_{n} E_{n}(t)+c_{-n} E_{-n}(t)\right) .
$$

The complex form of the $r$ th derivative of this sum, obtained by Sheng [4], is

$$
u_{n}^{(r)}(c, t)=s_{n}^{(r)}(c, t)-\left(c_{n} E_{n}^{(r)}(t)+c_{-n} E_{-n}^{(r)}(t)\right) .
$$

Received by the editors November 11, 1992 and, in revised form, August 25, 1994 and December 7, 1994.

1991 Mathematics Subject Classification. Primary 42A16, 42A20.

Key words and phrases. Modified complex trigonometric sums, weakly even sequences, $L^{1}$ convergence.

(C)1996 American Mathematical Society 
S. Kumari and B. Ram [2] introduced another set of modified cosine and sine sums as

$$
f_{n}(x)=\frac{a_{0}}{2}+\sum_{k=1}^{n} \sum_{j=k}^{n} \Delta\left(a_{j} / j\right) k \cos k x
$$

and

$$
g_{n}(x)=\sum_{k=1}^{n} \sum_{j=k}^{n} \Delta\left(a_{j} / j\right) k \sin k x .
$$

The complex form of the $r$ th derivative of these modified sums is

$$
g_{n}^{(r)}(c, t)=s_{n}^{(r)}(c, t)+\frac{i}{n+1}\left[c_{n+1} E_{n}^{(r+1)}(t)-c_{-(n+1)} E_{-n}^{(r+1)}(t)\right] .
$$

Remark 1. If $|n|^{r} c_{n} \rightarrow 0(|n| \rightarrow \infty)$, then $\left\|g_{n}^{(r)}-u_{n}^{(r)}\right\|_{1} \rightarrow 0(n \rightarrow \infty)$. Observe that by partial summation, we have

$$
E_{n}^{(r+1)}(t)=-i \sum_{k=0}^{n} E_{k}^{(r)}(t)+i(n+1) E_{n}^{(r)}(t)
$$

and similarly for $E_{-n}^{(r+1)}(t)$. Also from (1.3) we note that

$$
u_{n+1}^{(r)}(c, t)=s_{n}^{(r)}(c, t)-c_{n+1} E_{n}^{(r)}(t)-c_{-(n+1)} E_{-n}^{(r)}(t) .
$$

Hence,

$$
\begin{aligned}
u_{n+1}^{(r)}(c, t)-g_{n}^{(r)}(c, t)= & -c_{n+1} \frac{1}{n+1} \sum_{k=0}^{n} E_{k}^{(r)}(t) \\
& -c_{-(n+1)} \frac{1}{n+1} \sum_{k=1}^{n} E_{-k}^{(r)}(t) .
\end{aligned}
$$

If we assume $|n|^{r} c_{n} \rightarrow 0(|n| \rightarrow \infty)$, then by partial summation and the well-known properties of Fejér kernels, it follows that $\left\|g_{n}^{(r)}-u_{n}^{(r)}\right\|_{1} \rightarrow 0(n \rightarrow \infty)$.

Concerning the $L^{1}$-convergence of complex trigonometric series, F. Móricz [3] improved the result of Č. V. Stanojević and V. B. Stanojević [5] by assuming a weaker condition. The aim of this paper is to give sufficient conditions for the integrability of the $r$-times differentiated trigonometric series using complex trigonometric sums (1.3) and (1.4) and to obtain necessary and sufficient conditions for the $L^{1}$ convergence of the $r$ th derivative of the Fourier series. The case $r=0$ of our theorems yields the results of F. Móricz [3].

\section{RESUlts}

Let $1<p \leq 2$ be a real number. Denote by $q$ the conjugate exponent to $p$, i.e., $1 / p+1 / q=1$, by $I_{m}$ the dyadic interval $\left[2^{m-1}, 2^{m}\right)$ for $m \geq 1$, and by $\|\cdot\|_{1}$ the $L^{1}(-\pi, \pi)$-norm.

We prove the following theorems for the sum (1.3):

Theorem 1. Let $\left\{c_{k}\right\} \in W_{r}$ and

$$
\sum_{m=1}^{\infty} 2^{m(1 / q+r)}\left(\sum_{k \in I_{m}}\left|\Delta c_{k}\right|^{p}\right)^{1 / p}<\infty,
$$


for some $1<p \leq 2$ and $r \geq 0$. Then

(i) $\lim _{n \rightarrow \infty} s_{n}^{(r)}(c, t)=f^{(r)}(t)$ for all $0<|t| \leq \pi$,

(ii) $f^{(r)}(t) \in L^{1}(T)$ and $\left\|u_{n}^{(r)}(c)-f^{(r)}\right\|_{1}=o(1)$ as $n \rightarrow \infty$,

(iii) $\left\|s_{n}^{(r)}(f)-f^{(r)}\right\|_{1}=o(1)$ as $n \rightarrow \infty$ if and only if $|n|^{r} \hat{f}(n) \log |n|=o(1)$ as $|n| \rightarrow \infty$.

Theorem 2. Let $\left\{c_{k}\right\} \in W_{r}$ and

$$
\sum_{m=1}^{\infty} 2^{m(1 / q+r)}\left(\sum_{k \in I_{m}}\left|\Delta\left(c_{k}+c_{-k}\right)\right|^{p}\right)^{1 / p}<\infty,
$$

for some $1<p \leq 2$ and $r \geq 0$. Then statements (i)-(iii) of Theorem 1 hold.

Taking $r=0$ in Theorems 1 and 2 we obtain Theorems 3 and 2 of F. Móricz [3] respectively.

Considering the sums (1.4) instead of (1.3) and in view of the preceding Remark in Section 1, statement (ii) in Theorems 1 and 2 can be replaced by:

$\left(\mathrm{ii}^{\prime}\right) f^{(r)}(t) \in L^{1}(T)$ and $\left\|g_{n}^{(r)}(c)-f^{(r)}\right\|_{1} \rightarrow 0(n \rightarrow \infty)$.

Thus we have the following results:

Theorem 3. Under the hypothesis of Theorem 1, statements (i), (ii') and (iii) hold.

Theorem 4. Under the hypothesis of Theorem 2, statements (i), (ii') and (iii) hold.

\section{LEMMAS}

Lemma 1 (Sheng [4]). $\left\|D_{n}^{(r)}\right\|_{1}=(4 / \pi) n^{r} \log n+O\left(n^{r}\right), n \rightarrow \infty, r \in\{0,1,2, \ldots\}$.

Lemma 2 (Sheng [4]). $\left\|\widetilde{D}_{n}^{(r)}\right\|_{1}=O(1)\left(n^{r} \log n\right), n \rightarrow \infty, r \in\{0,1,2, \ldots\}$.

Lemma 3 (Sheng [4]). Let $r$ be a non-negative integer and $x \in[\pi / n, \pi]$, where $n \geq 1$. Then

$$
\begin{aligned}
D_{n}^{(r)}(x)= & \sum_{k=0}^{r-1} \frac{(n+1 / 2)^{k} \sin [(n+1 / 2) x+k \pi / 2]}{(\sin x / 2)^{r+1-k}} \Phi_{k}(x) \\
& +\frac{(n+1 / 2)^{r} \sin [(n+1 / 2) x+r \pi / 2]}{2 \sin x / 2}
\end{aligned}
$$

where each $\Phi_{k}$ denotes an appropriate bounded function dependent on $r$ but independent of $n$.

Lemma 4 (Sheng [4]). For each non-negative integer $n$,

$$
\left\|c_{n} E_{n}^{(r)}+c_{-n} E_{-n}^{(r)}\right\|_{1}=o(1), \quad n \rightarrow \infty
$$

holds if and only if $|n|^{r} c_{n} \log |n|=o(1),|n| \rightarrow \infty$, where $\left\{c_{n}\right\}$ is a complex sequence.

Lemma 5. Let $\left\{c_{k}\right\}$ be a sequence of complex numbers. Then for any $1<p \leq 2$ and $n \geq 1, r \geq 0$

$$
\frac{1}{n} \int_{0}^{\pi}\left|\sum_{k=n}^{2 n-1} c_{k} D_{k}^{(r)}(x)\right| d x \leq A_{p r} n^{r}\left(\frac{1}{n} \sum_{k=n}^{2 n-1}\left|c_{k}\right|^{p}\right)^{1 / p},
$$

where $A_{p r}$ is a constant depending upon $p$ and $r$. 
This lemma is an extension of Lemma 2.3 of Bojanic and Stanojević [1].

Proof. We write

$$
\begin{aligned}
\frac{1}{n} \int_{0}^{\pi}\left|\sum_{k=0}^{2 n-1} c_{k} D_{k}^{(r)}(x)\right| d x= & \frac{1}{n} \int_{0}^{\pi / n}\left|\sum_{k=n}^{2 n-1} c_{k} D_{k}^{(r)}(x)\right| d x \\
& +\frac{1}{n} \int_{\pi / n}^{\pi}\left|\sum_{k=n}^{2 n-1} c_{k} D_{k}^{(r)}(x)\right| d x \\
= & I_{1}+I_{2}, \quad \text { say. }
\end{aligned}
$$

Since $\left\|D_{k}^{(r)}\right\|_{1}=O\left(k^{r+1}\right)$, for the first integral in (3.2), we have

$$
I_{1}=O(1)\left\{n^{r-1} \sum_{k=n}^{2 n-1}\left|c_{k}\right|\right\},
$$

and now by Hölder's inequality, we have

$$
I_{1}=O(1)\left\{n^{r}\left(\frac{1}{n} \sum_{k-n}^{2 n-1}\left|c_{k}\right|^{p}\right)^{1 / p}\right\} .
$$

We now estimate $I_{2}$

$$
I_{2}=\frac{1}{n} \int_{\pi / n}^{\pi}\left|\sum_{k=n}^{2 n-1} c_{k} D_{k}^{(r)}(x)\right| d x .
$$

From Lemma 3, we have

$$
\begin{aligned}
I_{2}= & \frac{1}{n} \int_{\pi / n}^{\pi}\left|\sum_{k=n}^{2 n-1} c_{k}\left(\sum_{\lambda=0}^{r-1} \frac{(k+1 / 2)^{\lambda} \sin [(k+1 / 2) x+\lambda \pi / 2]}{(\sin x / 2)^{r+1-\lambda}} \Phi_{\lambda}\right)\right| d x \\
& +\frac{1}{n} \int_{\pi / n}^{\pi}\left|\sum_{k=n}^{2 n-1} c_{k} \frac{(k+1 / 2)^{r} \sin [(k+1 / 2) x+r \pi / 2]}{2 \sin x / 2}\right| d x \\
\leq & \Phi_{2 n-1}^{(1)}(x)+\Phi_{2 n-1}^{(2)}(x),
\end{aligned}
$$

where

$$
\begin{gathered}
\Phi_{2 n-1}^{(1)}(x)=\sum_{\lambda=1}^{r} \Phi_{2 n-1, \lambda}^{(1)}(x), \\
\Phi_{2 n-1, \lambda}^{(1)}(x)=\frac{1}{n} \int_{\pi / n}^{\pi}\left|\sum_{k=n}^{2 n-1} c_{k} \frac{(k+1 / 2)^{\lambda} \sin [(k+1 / 2) x+\lambda \pi / 2]}{(\sin x / 2)^{r+1-\lambda}} \Phi_{\lambda}(x)\right| d x .
\end{gathered}
$$

Since $\Phi_{\lambda}$ are bounded, it can be shown by Hölder's inequality that

$$
\begin{aligned}
\Phi_{2 n-1, \lambda}^{(1)}(x)=O(1) & \left\{\frac{1}{n}\left(\int_{\pi / n}^{\pi} \frac{d x}{(\sin x / 2)^{(r+1-\lambda) p}}\right)^{1 / p}\right. \\
& \left.\cdot\left(\int_{\pi / n}^{\pi}\left|\sum_{k=n}^{2 n-1} c_{k}(k+1 / 2)^{\lambda}[\sin (k+1 / 2) x+\lambda \pi / 2]\right|^{q} d x\right)^{1 / q}\right\} .
\end{aligned}
$$


Since

$$
\begin{aligned}
\int_{\pi / n}^{\pi} \frac{d x}{(\sin x / 2)^{(r+1-\lambda) p}} & \leq \pi^{(r+1-\lambda) p} \int_{\pi / n}^{\pi} \frac{d x}{x^{(r+1-\lambda) p}} \\
& \leq \frac{\pi}{(r+1-\lambda) p-1} n^{(r+1-\lambda) p-1},
\end{aligned}
$$

it follows that

$$
\begin{aligned}
\Phi_{2 n-1, \lambda}^{(1)}(x) \leq & \left(\frac{\pi}{(r+1-\lambda) p-1}\right)^{1 / p} n^{r-\lambda-1 / p} \\
& \cdot\left(\int_{0}^{\pi}\left|\sum_{k=n}^{2 n-1} c_{k}\left(k+\frac{1}{2}\right)^{\lambda} \sin [(k+1 / 2) x+\lambda \pi / 2]\right|^{q} d x\right)^{1 / q} .
\end{aligned}
$$

Now, by using Hausdorff-Young inequality, we get

$$
\begin{gathered}
\left(\frac{1}{\pi} \int_{0}^{\pi}\left|\sum_{k=n}^{2 n-1} c_{k}(k+1 / 2)^{\lambda} \sin [(k+1 / 2) x+\lambda \pi / 2]\right|^{q} d x\right)^{1 / q} \\
\quad \leq 2\left(\sum_{k=n}^{2 n-1}\left|c_{k}(k+1 / 2)^{\lambda}\right|^{p}\right)^{1 / p} \leq 2^{1+\lambda} n^{\lambda}\left(\sum_{k=n}^{2 n-1}\left|c_{k}\right|^{p}\right)^{1 / p} .
\end{gathered}
$$

Thus,

$$
\Phi_{2 n-1, \lambda}^{(1)}(x) \leq A_{p r}^{(0)} n^{r}\left(\frac{1}{n} \sum_{k=n}^{2 n-1}\left|c_{k}\right|^{p}\right)^{1 / p}
$$

Therefore

$$
\Phi_{2 n-1}^{(1)}(x) \leq A_{p r}^{(1)} n^{r}\left(\frac{1}{n} \sum_{k=n}^{2 n-1}\left|c_{k}\right|^{p}\right)^{1 / p}
$$

Similarly,

$$
\Phi_{2 n-1}^{(2)}(x) \leq A_{p r}^{(2)} n^{r}\left(\frac{1}{n} \sum_{k=n}^{2 n-1}\left|c_{k}\right|^{p}\right)^{1 / p}
$$

Combining the above estimates, we get

$$
I_{1}+I_{2} \leq A_{p r} n^{r}\left(\frac{1}{n} \sum_{k=n}^{2 n-1}\left|c_{k}\right|^{p}\right)^{1 / p} .
$$

This completes the proof of Lemma 5 .

Lemma 6. Let $r$ be a non-negative integer and $0<\varepsilon<\pi$. Then there exists $A_{r \varepsilon}>0$ such that for all $\varepsilon \leq|t| \leq \pi$ and all $n \geq 1$,

$$
\left|E_{n}^{(r)}(t)\right|,\left|E_{-n}^{(r)}(t)\right| \leq A_{r \varepsilon} n^{r} /|t|
$$

and

$$
\left|D_{n}^{(r)}(t)\right|,\left|\widetilde{D}_{n}^{(r)}(t)\right| \leq 2 A_{r \varepsilon} n^{r} /|t| .
$$


Proof. The case $r=0$ is trivial. For $r \geq 1$, we have

$$
-i^{r} E_{n}^{(r)}(t)=\sum_{k=0}^{n} k^{r} e^{i k t}=\sum_{k=0}^{n}\left(\Delta k^{r}\right) E_{k}(t)+(n+1)^{r} E_{n}(t),
$$

and so

$$
\left|E_{n}^{r}(t)\right| \leq\left(A_{0 \varepsilon} /|t|\right)\left\{\left(\sum_{k=0}^{n}\left|\Delta k^{r}\right|\right)+(n+1)^{r}\right\} \leq A_{r \varepsilon} n^{r} /|t|
$$

for some constant $A_{r \varepsilon}$. Since

$$
E_{-n}^{(r)}(t) \mid=(-1)^{r} E_{n}^{(r)}(-t),
$$

we obtain $\left|E_{-n}^{(r)}(t)\right| \leq A_{r \varepsilon} n^{r} /|t|$. The other two inequalities follow from the equations (1.1) and (1.2).

\section{Proofs of the theorems}

Proof of Theorem 1. Performing Abel's transformation on the $r$ th derivative of the partial sums of a general trigonometric series, it is easy to see that

$$
\begin{aligned}
s_{n}^{(r)}(c, t)= & \sum_{k=0}^{n-1} \Delta c_{k} D_{k}^{(r)}(t)+\sum_{k=0}^{n-1} \Delta\left(c_{-k}-c_{k}\right) E_{-k}^{(r)}(t) \\
& +c_{n} E_{n}^{(r)}(t)+c_{-n} E_{-n}^{(r)}(t) .
\end{aligned}
$$

By the use of Lemma 6 and Hölder's inequality, we get

$$
\begin{aligned}
\sum_{k=1}^{\infty}\left|\Delta c_{k} D_{k}^{(r)}(t)\right| & \leq \lim _{n \rightarrow \infty} \frac{A_{r+1}}{|t|}\left(\sum_{k=1}^{n} k^{r}\left|\Delta c_{k}\right|\right) \\
& =\lim _{m \rightarrow \infty} \frac{A_{r+1}}{|t|} \sum_{j=1}^{m}\left(\sum_{k=2^{j-1}}^{2^{j}-1} k^{r}\left|\Delta c_{k}\right|\right) \quad \text { for } n=2^{m}-1 \\
& =\lim _{m \rightarrow \infty} \frac{A_{r+1}}{|t|} \sum_{j=1}^{m} 2^{j(1 / q+r)}\left(\sum_{k \in I_{j}}\left|\Delta c_{k}\right|^{p}\right)^{1 / p}<\infty
\end{aligned}
$$

and

$$
\begin{aligned}
\sum_{k=3}^{\infty}\left|\Delta\left(c_{-k}-c_{k}\right)\right|\left|E_{-k}^{(r)}(t)\right| & \leq \frac{A_{r+1}}{|t|}\left\{\sum_{k=3}^{\infty} k^{r}\left|\Delta\left(c_{-k}-c_{k}\right)\right|\right\} \\
& \leq \frac{A_{r+1}}{|t|}\left\{\sum_{k=3}^{\infty} k^{r} \log k\left|\Delta\left(c_{-k}-c_{k}\right)\right|\right\}<\infty
\end{aligned}
$$

where $A_{r+1}$ is a suitable constant. Also $\lim _{n \rightarrow \infty}\left\{c_{n} E_{n}^{(r)}(t)+c_{-n} E_{-n}^{(r)}(t)\right\}=0$ for $0<$ $|t| \leq \pi$, as $\left\{c_{k}\right\}$ is a null sequence. These imply that $f^{(r)}(t)=\sum_{k=1}^{\infty} \Delta c_{k} D_{k}^{(r)}(t)+$ $\sum_{k=1}^{\infty} \Delta\left(c_{-k}-c_{k}\right) E_{-k}^{(r)}(t)$ exists for $0<|t| \leq \pi$, and thus the proof of (i) is completed. 
Furthermore, from the above and (1.3), for $t \neq 0$, we have

$$
f^{(r)}(t)-u_{n}^{(r)}(c, t)=\sum_{k=n}^{\infty} \Delta c_{k} D_{k}^{(r)}(t)+\sum_{k=n}^{\infty} \Delta\left(c_{-k}-c_{k}\right) E_{-k}^{(r)}(t) .
$$

This implies that

$$
\left\|f^{(r)}-u_{n}^{(r)}(c)\right\|_{1} \leq\left\|\sum_{k=n}^{\infty} \Delta c_{k} D_{k}^{(r)}\right\|_{1}+\sum_{k=n}^{\infty}\left|\Delta\left(c_{-k}-c_{k}\right)\right|\left\|E_{-k}^{(r)}\right\|_{1} .
$$

Lemma 1, Lemma 2 and Lemma 5 imply that

$$
\begin{aligned}
\left\|f^{(r)}-u_{n}^{(r)}(c)\right\|_{1} \leq & A_{p r} \sum_{m=j}^{\infty} 2^{m(1 / q+r)}\left(\sum_{k \in I_{m}}\left|\Delta c_{k}\right|^{p}\right)^{1 / p} \\
& +O\left(\sum_{k=n}^{\infty}\left|\Delta\left(c_{-k}-c_{k}\right)\right| k^{r} \log k\right)=o(1), \quad n \rightarrow \infty
\end{aligned}
$$

by the hypothesis of the theorem; here $j=j(n)$ denotes the integer for which $2^{j-1} \leq n<2^{j}$. Since $u_{n}^{(r)}(c, t)$ is a polynomial, it follows that $f^{(r)} \in L^{1}(T)$, which proves assertion (ii).

We further notice that

$$
\begin{aligned}
\left\|f^{(r)}-s_{n}^{(r)}(f)\right\|_{1} & =\left\|f^{(r)}-u_{n}^{(r)}(c)+u_{n}^{(r)}(c)-s_{n}^{(r)}(f)\right\|_{1} \\
& \leq\left\|f^{(r)}-u_{n}^{(r)}\right\|_{1}+\left\|u_{n}^{(r)}(c)-s_{n}^{(r)}(f)\right\|_{1} \\
& =\left\|f^{(r)}-u_{n}^{(r)}(c)\right\|_{1}+\left\|\hat{f}(n) E_{n}^{(r)}+\hat{f}(-n) E_{-n}^{(r)}\right\|_{1}
\end{aligned}
$$

and

$$
\begin{aligned}
\left\|\hat{f}(n) E_{n}^{(r)}+\hat{f}(-n) E_{-n}^{(r)}\right\|_{1} & =\left\|u_{n}^{(r)}(c)-s_{n}^{(r)}(f)\right\|_{1} \\
& \leq\left\|f^{(r)}-s_{n}^{(r)}(f)\right\|_{1}+\left\|f^{(r)}-u_{n}^{(r)}(c)\right\|_{1} .
\end{aligned}
$$

Since $\left\|f^{(r)}-u_{n}^{(r)}(c)\right\|_{1}=o(1), n \rightarrow \infty$, by (ii), and $\left.\| \hat{f}(n) E_{n}^{(r)}+\hat{f}(-n) E_{-n}^{(r)}\right) \|_{1}=o(1)$, $n \rightarrow \infty$, if and only if $n^{r} \hat{f}(n) \log n=o(1),|n| \rightarrow \infty$, by Lemma 4 , then assertion (iii) follows.

Proof of Theorem 2. As before, an application of Abel's transformation yields

$$
\begin{aligned}
s_{n}^{(r)}(c, t)= & \sum_{k=0}^{n-1} \Delta c_{k} D_{k}^{(r)}(t)+\sum_{k=0}^{n-1} \Delta\left(c_{-k}-c_{k}\right) E_{-k}^{(r)}(t) \\
& +c_{n} E_{n}^{(r)}(t)+c_{-n} E_{-n}^{(r)}(t) .
\end{aligned}
$$

Then making use of (1.1) and (1.2), we get

$$
\begin{aligned}
s_{n}^{(r)}(c, t)= & \frac{1}{2} \sum_{k=0}^{n-1} \Delta\left(c_{k}+c_{-k}\right) D_{k}^{(r)}(t)+i \sum_{k=0}^{n-1} \Delta\left(c_{-k}-c_{k}\right) \widetilde{D}_{k}^{(r)}(t) \\
& +c_{n} E_{n}^{(r)}(t)+c_{-n} E_{-n}^{(r)}(t) .
\end{aligned}
$$

The rest of the arguments are similar to the proof of Theorem 1, and therefore we omit them. 
Proofs of Theorems 3 and 4. We observe that under the assumptions of Theorems 1 and $2,\left\{c_{k}\right\} \in W_{r}$ and (2.1), respectively (2.2). Thus $\sum_{|k|=n}^{\infty}|k|^{r}\left|\Delta c_{k}\right| \rightarrow 0(n \rightarrow$ $\infty)$, which together with $c_{n} \rightarrow 0(|n| \rightarrow \infty)$ implies that $|n|^{r} c_{n} \rightarrow 0(|n| \rightarrow \infty)$. Hence, by Remark of Section 1, Theorems 3 and 4 follow.

\section{Conclusions}

1. A sequence $\left\{c_{k}\right\}$ of complex numbers is said to belong to the class $S_{p \alpha r}^{*}$ of Sheng [4] if

$$
\left\{c_{k}\right\} \in W_{r}
$$

(5.2) there exists a sequence $\left\{A_{k}\right\}$ of positive numbers such that

$$
A_{k} \curvearrowleft 0 \text { and } \quad \sum_{k=1}^{\infty} k^{\alpha} A_{k}<\infty \quad \text { for some } \alpha \geq 0
$$

and

$$
\frac{1}{n^{p(\alpha-r)+1}} \sum_{k=1}^{n} \frac{\left|\Delta c_{k}\right|^{p}}{A_{k}^{p}}=O(1) \quad \text { for some } \alpha \geq 0,1<p \leq 2 .
$$

Conditions (5.2) and (5.3) imply (2.1). In fact, by (5.3) and quasi-monotonicity of $\left\{A_{k}\right\}$,

$$
\left(\sum_{k \in I_{m}}\left|\Delta c_{k}\right|^{p}\right)^{1 / p} \leq K 2^{m / p} 2^{m(\alpha-r)} A_{2^{m-1}}
$$

with an absolute constant $K>0$. Hence,

$$
\begin{aligned}
\sum_{m=1}^{\infty} 2^{m(1 / q+r)}\left(\sum_{k \in I_{m}}\left|\Delta c_{k}\right|^{p}\right)^{1 / p} & \leq K \sum_{m=1}^{\infty} 2^{m / p+m / q} 2^{m \alpha} A_{2^{m-1}} \\
& =2^{1+\alpha} K \sum_{m=8}^{\infty} 2^{m} 2^{m \alpha} A_{2^{m}}<\infty \quad \text { due to (5.2) }
\end{aligned}
$$

Therefore Theorem 1 implies the following:

Theorem A (Sheng [4]). Let $\left\{c_{k}\right\} \in S_{\text {par }}^{*}, \alpha \geq 0$ and $r \in\{0,1,2, \ldots,[\alpha]\}$. Then, for $t \neq 0$

(i) $\lim _{n \rightarrow \infty} s_{n}^{(r)}(c, t)=f^{(r)}(t)$,

(ii) $f^{(r)}(t) \in L^{1}(T)$

(iii) $\left\|s_{n}^{(r)}(f)-f^{(r)}\right\|_{1}=o(1)$ as $n \rightarrow \infty$ if and only if $|n|^{r} \hat{f}(n) \log |n|=o(1)$ as $|n| \rightarrow \infty$.

2. The following examples show that Theorems 1 and 2 are not comparable to one another.

Example 1. Let a null sequence $\left\{c_{k}\right\}$ be defined by $\Delta c_{k}=1 / 2^{m r} m^{3}$ for $k=2^{m}$, $\Delta c_{k}=-1 / 2^{m r} m^{3}$ for $k=-2^{m}$ with $m \geq 0$, and let $\Delta c_{k}=0$ otherwise. Then, $\sum_{k=1}^{\infty}\left|\Delta\left(c_{k}-c_{-k}\right)\right| k^{r} \log k<\infty$ and (2.2) is satisfied, but condition (2.1) is not satisfied. Thus, only Theorem 2 applies in this case. 
Example 2. Let a null sequence $\left\{c_{k}\right\}$ be defined by $\Delta c_{k}=1 / k^{r+2}$ for $k \geq 1$, $\Delta c_{k}=1 / 2^{m r} m^{3}$ for $k=-2^{m}$ with $m \geq 0$ and $\Delta c_{k}=0$ otherwise. Then, $\sum_{k=1}^{\infty}\left|\Delta\left(c_{k}-c_{-k}\right)\right| k^{r} \log k<\infty$ and (2.1) is satisfied, but condition (2.2) is not satisfied. Thus, only Theorem 1 applies in this case.

\section{ACKNOWLEDGMENT}

The authors are thankful to the referee for his wise comments which have definitely improved the representation of the paper.

\section{REFERENCES}

1. R. Bojanic and Č. V. Stanojević, A class of $L^{1}$-convergence, Trans. Amer. Math. Soc. 269 (1982), 677-683. MR 83e:42004

2. S. Kumari and B. Ram, $L^{1}$-convergence of a modified cosine sum, Indian J. Pure Appl. Math. 19 (1988), 1101-1104. MR 90g:42010

3. F. Móricz, On integrability and $L^{1}$-convergence of complex trigonometric series, Proc. Amer. Math. Soc. 113 (1991), 53-64. MR 91k:42002

4. Sheng Shu Yun, The extension of the theorem of $\check{C}$. V. Stanojević and V. B. Stanojević, Proc. Amer. Math. Soc. 110 (1990), 895-905.

5. C. V. Stanojević and V. B. Stanojević, Generalisations of Sidon-Telyakovskiu theorem, Proc. Amer. Math. Soc. 101 (1987), 679-684. MR 89a:42017

Department of Mathematics, Maharshi Dayanand University, Rohtak-124001, India 